\title{
Advanced time domain wave-number sensing for structural acoustic systems. II. Active radiation control of a simply supported beam
}

\author{
J. P. Maillard and C. R. Fuller \\ Vibration and Acoustics Laboratories, Mechanical Engineering Department, Virginia Polytechnic Institute \\ and State University, Blacksburg, Virginia 24061-0238
}

(Received 9 June 1993; revised 21 October 1993; accepted 4 February 1994)

\begin{abstract}
A real time structural acoustic sensor and associated signal processing is developed and applied to the active control of sound radiated by a simply supported beam. The sensor consists of multiple accelerometers mounted on the structure. An array of FIR filters processes the measured structural information to provide an estimate of the structural wave-number component coupled to acoustic radiation in a prescribed direction. This time domain signal is used as the error information in a feedforward adaptive control approach. The single channel filtered-X LMS algorithm is implemented here. Computer simulations in the discrete time domain demonstrate the ability of the sensor to replace the use of error microphones in the far field. The described sensor represents a significant alternative to the use of distributive structural sensors (for example piezoelectric material) by providing accurate radiation information over a broadband frequency range.
\end{abstract}

PACS numbers: $43.40 . \mathrm{At}, 43.40 . \mathrm{Cw}, 43.40 . \mathrm{Vn}, 43.60 . \mathrm{Gk}$

\section{INTRODUCTION}

Sound radiated by vibrating structures is an important issue in numerous industrial applications and much research has been conducted in the field of noise control. In this respect, active structural active control (ASAC) techniques have been successfully applied in situations where passive methods are inefficient, i.e., in the low-frequency range. Previously, Fuller has demonstrated both theoretically ${ }^{1}$ and experimentally ${ }^{2}$ that sound radiated from structures can be controlled by applying vibrating point forces directly to the structure, while the error information is taken from microphones in the acoustic far field. More recent work has been devoted to the development of new actuators (piezoelectric ceramics) and distributed structural sensors (PVDF films) designed to eliminate the use of far-field microphones and achieve global control. In particular, Clark and Fuller ${ }^{3}$ have demonstrated the use of PVDF film modal sensors in ASAC approaches applied to a simply supported plate under single frequency excitation. Also, Fuller and Burdisso ${ }^{4}$ recently suggested the use of wavenumber cost function in ASAC.

In a companion paper, ${ }^{5}$ the authors theoretically introduced a sensing approach using point structural sensors in parallel with an array of digital filters. This method provides estimates of the structural wave-number component(s) coupled to acoustic radiation in prescribed direction(s) for finite planar radiators. The sensor implements a number of accelerometers equally spaced on the structure. The accelerometer outputs are passed through finite impulse response (FIR) filters and summed to provide farfield radiation information. This time domain signal can be directly used as the error information in control algorithms. One of the main advantages of the method is that it provides radiation information over a broadband frequency range and can thus be applied to randomly excited structures. It will be referred to as real time structural acoustic sensing.

The present work illustrates structural acoustic sensing applied to the active control of acoustic radiation from a simply supported beam. The control approach is the time domain adaptive filtered-X LMS algorithm and a single control input is applied to the structure in order to minimize the far-field pressure in one direction. Computer simulations are performed in the discrete time domain to demonstrate the use of the sensing procedure.

Recalling the theoretical developments of the companion paper, ${ }^{5}$ the sensor is described for the particular case of a simply supported beam in the first part of the paper. The influence of structural acoustic sensing on the control performance is first studied in the frequency domain. In this case, theoretical transfer functions are used to obtain the optimal control input. Therefore, the system does not take into account the causality issue and neglect the errors introduced by the use of digital filters that have a discrete impulse response. To obtain more realistic results, timedomain simulations are performed using discrete impulse responses for the structural acoustic system. In the second part, the filtered-X version of the Widrow-Hoff LMS algorithm ${ }^{6}$ is briefly introduced and the computer simulation procedure is discussed along with the design of the filter array for the sensor. Finally, the third part of the paper presents results from the control simulations in the case of broadband excitations encompassing the first four bending modes of the beam.

\section{SENSOR DESCRIPTION}

The sensing technique presented in the companion paper is applied here to the case of a baffled simply supported 


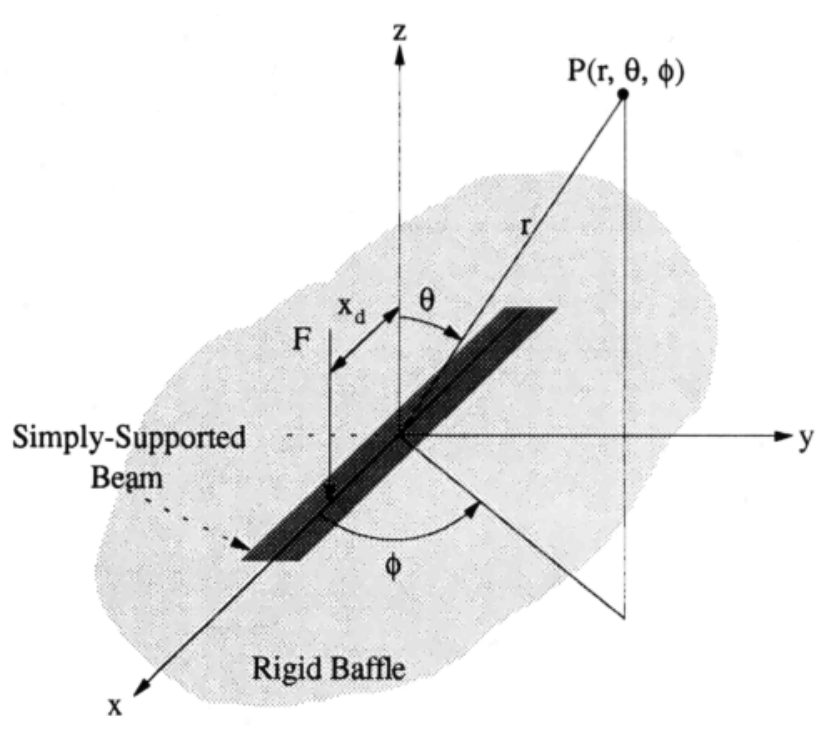

FIG. 1. Coordinate system of the simply supported beam.

beam. With the purpose of establishing basic concepts and notations, the equations governing the beam response and its acoustic radiation in the far field are first recalled along with the desired transfer functions to be modeled in the sensor implementation. The sensor is then further described in the frequency domain in terms of control performance; the acoustic radiation of the beam after control is presented for both cases where continuous and estimated wavenumber components are used as the error information.

\section{A. Simply supported beam system}

Figure 1 shows an infinite baffled simply supported beam of length $L_{x}$ and width $L_{y}$ excited by a harmonic point force $f^{d}(t)=F^{d} e^{j \omega t}$ at point $x=x_{d} ; \omega$ represents the angular frequency, $t$ is the continuous time, and $F^{d}$ is the disturbance complex amplitude. The beam response is assumed to have constant amplitude of motion in the $y$ direction and the radiated acoustic field is investigated in the $x-z$ plane $(\phi=0)$. The surrounding media is air and is assumed to have negligible influence on the in vacuo structural response.

\section{Structural response}

The beam out-of-plane displacement $w(x, t)$ can be given as a linear combination of the modes as

$$
w(x, t)=\sum_{p=1}^{\infty} q_{p}(\omega) \psi_{p}(x) e^{j \omega t}, \quad-\frac{L_{x}}{2} \leqslant x \leqslant \frac{L_{x}}{2},
$$

where $q_{p}(\omega)$ is the $p$ th modal displacement and $\psi_{p}(x)$ the $p$ th eigenfunction. Using the coordinate system shown in Fig. 1, the eigenfunctions for the simply supported boundary condition are given by

$$
\psi_{p}(x)=(2 / m)^{1 / 2} \sin \left[\gamma_{p}\left(x+L_{x} / 2\right)\right],
$$

where $\gamma_{p}=p \pi / L_{x}$. The factor $(2 / m)^{1 / 2}$ results from the normalization chosen for the eigenfunctions, such that they are orthonormal with respect to the mass $m$ of the beam.
The modal displacement $q_{p}(\omega)$ is defined as

$$
\begin{aligned}
q_{p}(\omega) & =H_{p}(\omega) \psi_{p}\left(x_{d}\right) F^{d} \\
& =\frac{1}{\omega_{p}^{2}-\omega^{2}+2 j \eta_{p} \omega_{p} \omega} \psi_{p}\left(x_{d}\right) F^{d},
\end{aligned}
$$

where $H_{p}(\omega)$ is the $p$ th modal frequency response function; $\omega_{p}$ and $\eta_{p}$ are the $p$ th natural frequency and modal damping ratio, respectively. The analytical natural frequencies are

$$
\omega_{p}^{2}=\gamma_{p}^{4}\left(E I / \rho_{s} S_{s}\right),
$$

where $\rho_{s}$ is the beam density, $S_{s}$ the beam section area, and $E I$ the bending stiffness.

The beam response due to the input disturbance, $f^{d}(t)=F^{d} e^{j \omega t}$, is then controlled with two piezoelectric patches bonded symmetrically to the front and back surface of the beam and driven $180^{\circ}$ out-of-phase. The corresponding excitation is modeled by a line bending moment pair with complex amplitude $M^{c}$ located at the end of the patches. ${ }^{7}$ The beam response due to the control input is obtained by replacing in Eq. (3) the point force excitation term $\psi_{p}\left(x_{d}\right) F^{d}$ by a bending moment excitation term $m_{p} M^{c}$. The control modal force component $m_{p}$ can be shown to be given by

$$
m_{p}=\frac{d}{d x}\left\{\psi_{p}\left(x_{c}+c_{a}\right)-\psi_{p}\left(x_{c}-c_{a}\right)\right\}
$$

$x_{c}$ is the coordinate of the center of the actuator, and $2 c_{a}$ is the actuator length. From the superposition principle, the controlled system response is the sum of the response due to the disturbance input and the response due to the control input. The modal displacement of the controlled system $q_{p}^{c}(\omega)$ becomes

$$
q_{p}^{c}(\omega)=H_{p}(\omega)\left[\psi_{p}\left(x_{d}\right) F^{d}+m_{p} M^{c}\right] .
$$

Finally, the acceleration distribution is the second partial derivative with respect to time of the displacement response, i.e.,

$$
\ddot{w}(x, t)=\ddot{W}(x) e^{j \omega t}=-\omega^{2} W(x) e^{j \omega t}, \quad-\frac{L_{x}}{2} \leqslant x \leqslant \frac{L_{x}}{2} .
$$

\section{Radiation in the acoustic far field}

The far-field pressure $p(r, \theta, t)$ radiated by the beam at point $(r, \theta, \phi=0)$ can be expressed in terms of structural information as ${ }^{8}$

$$
p(r, \theta, t)=\frac{\rho e^{-j k_{0} r}}{2 \pi r} \tilde{\ddot{W}}\left(k_{0} \sin \theta\right) e^{j \omega t},
$$

where $k_{0}=\omega / c$ is the acoustic wave number, $c$ the speed of sound in the medium, and $\rho$ is its density. In the above expression, $\tilde{\tilde{W}}\left(k_{0} \sin \theta\right)$ denotes the wave-number transform of the beam normal acceleration distribution $\ddot{W}(x)$ defined in Eq. (7). It can be written as the spatial Fourier transform of the beam response. Since the model assumes a constant response versus the $y$ direction, the structural waves travel solely along the $x$ direction and the structural 
wave number in the $y$ direction is zero, i.e., $k_{y}=0$. Therefore, the beam acceleration wave-number transform simplifies as

$$
\tilde{W}\left(k_{x}\right)=L_{y} \int_{-L_{x} / 2}^{L_{x} / 2} \ddot{W}(x) e^{j k_{x} x} d x .
$$

Substituting Eqs. (7) and (1), Eq. (9) can be rewritten as

$$
\tilde{\ddot{W}}\left(k_{x}\right)=-\omega^{2} \sum_{p=1}^{\infty} q_{p}(\omega) \xi_{p}\left(k_{x}\right),
$$

where

$$
\xi_{p}\left(k_{x}\right)=L_{y} \int_{-L_{x} / 2}^{L_{x} / 2} \psi_{p}(x) e^{j k_{x} x} d x .
$$

Here, $\xi_{p}\left(k_{x}\right)$ is defined as the wave-number transform of the $p$ th eigenfunction and will be referred to as the $p$ th modal wave-number component. A closed-form expression of the above formulation can be found in the Appendix of the companion paper. ${ }^{5}$

Note that Eq. (8) is only valid in the far field. This representation is equivalent to the stationary phase approximation discussed by Junger and Feit. ${ }^{8}$ As seen from Eq. (8), the radiated far-field pressure is solely a function of the wave-number component evaluated at wave numbers $k_{x}=k_{0} \sin \theta$ that are smaller than the acoustic wave number $k_{0}$ by definition of the sine function. In other words, only the structural motion with supersonic wave numbers $k_{x} \leqslant k_{0}$ radiates in the far field. Moreover, it is straightforward to show from $\mathrm{Eq}$. (8) that the supersonic wavenumber component $\widetilde{\ddot{w}}\left(k_{0} \sin \theta, t\right)$ is proportional to the time-shifted far-field pressure $p(r, \theta, t+r / c)$, i.e., it completely describes the system in terms of radiated energy in the far field. The sensor configuration described in the following section is based on the estimate of the wave-number component corresponding to a prescribed direction of radiation $\theta$ in the $x-z$ plane.

\section{B. Sensor configuration}

The governing equations for the sensor are presented in the case of the above simply supported beam system. The wave-number information is approximated by the summation of the structural acceleration measured at a number of points and multiplied in the frequency domain by complex transfer functions.

\section{Discrete wave-number transform}

The structural acceleration is measured at locations $x_{i}=-L_{x} / 2+\Delta x / 2(2 i-1), i=1,2, \ldots, N_{d}$ by means of $N_{d}$ accelerometers. The spacing of the accelerometers is given by $\Delta x=L_{x} / N_{d}$. The structural wave-number component in Eq. (9) is approximated by a discrete expression defined as

$$
\tilde{\ddot{W}}_{d}\left(k_{x}\right)=L_{y} \Delta x \sum_{i=1}^{N_{d}} \ddot{W}\left(x_{i}\right) e^{j k_{x} x_{i}} .
$$

Note that the accuracy of the discrete wave-number transform depends on both the number of point sensors $N_{d}$ and the frequency $\omega$ due to the fact that the structural response becomes more complicated at higher frequencies in terms of spatial variation. For instance, results from the companion paper ${ }^{5}$ show that a four-point sensor $\left(N_{d}=4\right)$ gives a fairly good approximation over the first four bending modes of a simply supported beam. Analogous to the continuous representation, Eq. (12) is expressed in terms of modal contributions as

$$
\tilde{\ddot{W}}_{d}\left(k_{x}\right)=-\omega^{2} \sum_{p=1}^{\infty} q_{p}(\omega) \xi_{p}^{d}\left(k_{x}\right),
$$

where the discrete modal wave-number components are given by

$$
\xi_{p}^{d}\left(k_{x}\right)=L_{y} \Delta x \sum_{i=1}^{N_{d}} \psi_{p}\left(x_{i}\right) e^{j k_{x} x_{i}}
$$

Introducing the time dependence $e^{j \omega t}$ and the angular frequency $\omega=k_{0} c$ in Eq. (12) yields

$$
\tilde{\ddot{w}}_{d}\left(k_{x}, t\right)=\sum_{i=1}^{N_{d}} H_{i}(\omega) \ddot{w}\left(x_{i}, t\right),
$$

where the transfer functions $H_{i}(\omega)$ to be modeled by the array of filters are written as

$$
H_{i}(\omega)=L_{y} \Delta x e^{j \omega \tau_{i}}, \quad i=1,2, \ldots, N_{d},
$$

$\tau_{i}=\left(x_{i} / c\right) \sin \theta$ is a time delay, characteristic of each acoustic path. The transfer functions in Eq. (16) have a constant magnitude and a linear phase term. Therefore, finite impulse response (FIR) filters will be used in the computer simulations discussed in the next part as well as in the practical implementation of the technique. Note that $\tau_{i}$ takes both positive and negative values. Recalling the time convention $e^{j \omega t}$, only negative values of $\tau_{i}$ ensure the transfer function $H_{i}(\omega)$ to be causal.

\section{Modifled transfer functions}

In order to obtain causal transfer functions and optimize the order of the filters, a time delay $\Delta \tau$ is introduced in Eq. (16). The modified transfer functions become

$$
\bar{H}_{i}(\omega)=L_{y} \Delta x e^{j \omega \bar{\tau}_{i}}, \quad i=1,2, \ldots, N_{d},
$$

where $\bar{\tau}_{i}=\tau_{i}-\Delta \tau$. The sensor output is now $\bar{e}(t)=e(t$ $-\Delta \tau)=\widetilde{\ddot{w}}_{d}\left(k_{x}, t-\Delta \tau\right)$. It has been shown in the companion paper ${ }^{5}$ that this modified error signal yields the same control performances as the error signal without delay, $e(t)=\widetilde{\ddot{w}}_{d}\left(k_{x}, t\right)$, when used in a feedforward control approach. Taking $\Delta \tau=\max \left\{\tau_{i}\right\}$ ensures causal frequency response functions $\left(\bar{\tau}_{i} \leqslant 0, i=1,2, \ldots, N_{d}\right)$ and makes one transfer function equal to unity, i.e., the number of filters to design is now $N_{d}-1$. Moreover, the modified delays $\bar{\tau}_{i}$ are minimized which optimizes the order of the filters. More details on the above discussion can be found in the companion paper.

\section{Control performance}

As suggested earlier, the discrete wave-number component $\widetilde{\ddot{w}}_{d}\left(k_{x}, t\right)$ varies from its continuous representation $\widetilde{w}\left(k_{x}, t\right)$ depending on the number of point sensors $N_{d}$ and the driving frequency $\omega$ (Ref. 5). In terms of radiation 
control, it is of interest to analyze the influence of these variations on the controller performance. In this section, the radiation control of a simply supported beam is analytically investigated in the frequency domain over its first four bending modes using both types of error information, i.e., continuous and discrete wave-number components corresponding to radiation in a prescribed direction.

\section{Optimal control force}

For a single channel feedforward control structure, the optimum complex amplitude of the control force $M^{c}$ is defined so as to minimize a quadratic cost function $J\left(M^{c}\right)$ of the error information. Here, the error information is the time-varying wave-number component associated with the direction of radiation $\theta$. Therefore, the cost function can be expressed as the mean-square value of the error signal, i.e.,

$$
J\left(M^{c}\right)=\tilde{\ddot{w}}\left(k_{0} \sin \theta, t\right) \tilde{\ddot{w}} *\left(k_{0} \sin \theta, t\right)
$$

for the continuous representation and

$$
J_{d}\left(M^{c}\right)=\tilde{\ddot{w}}_{d}\left(k_{0} \sin \theta, t-\Delta \tau\right) \tilde{\ddot{w}}_{d}^{*}\left(k_{0} \sin \theta, t-\Delta \tau\right)
$$

for the discrete representation, where $\tilde{\ddot{w}} *$ denotes the complex conjugate of $\tilde{\ddot{w}}$. Both $J\left(M^{c}\right)$ and $J_{d}\left(M^{c}\right)$ are quadratic functions of the control complex amplitude $M^{c}$. Therefore, they present only one minimum. The corresponding optimum control force is found by taking the partial derivatives of the cost function with respect to the real and imaginary parts of the control force. Setting these two equations to zero yields

$$
M^{c}=G(\omega) F^{d}=-\frac{\sum_{p=1}^{\infty} H_{p}(\omega) \psi_{p}\left(x_{d}\right) \xi_{p}\left(k_{x}\right)}{\sum_{p=1}^{\infty} H_{p}(\omega) m_{p} \xi_{p}\left(k_{x}\right)} F^{d}
$$

for the continuous representation and

$$
M_{d}^{c}=G_{d}(\omega) F^{d}=-\frac{\sum_{p=1}^{\infty} H_{p}(\omega) \psi_{p}\left(x_{d}\right) \xi_{p}^{d}\left(k_{x}\right)}{\sum_{p=1}^{\infty} H_{p}(\omega) m_{p} \xi_{p}^{d}\left(k_{x}\right)} F^{d}
$$

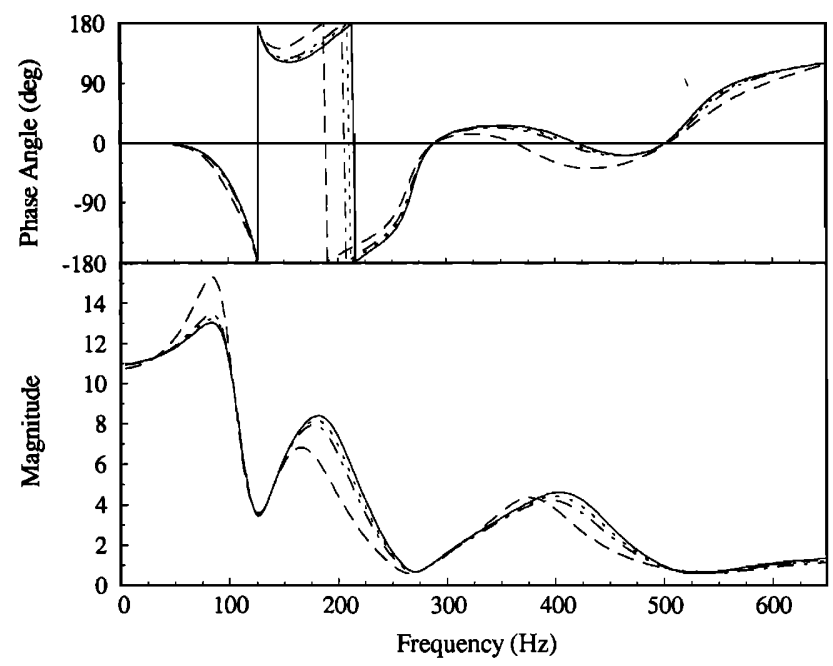

FIG. 2. Optimal controller frequency response function based on (a) - , continuous wave-number component; (b) --, three; (c) - - -, five; and (d)......., seven point sensors.
TABLE I. Beam characteristics.

\begin{tabular}{ll}
\hline \hline Length & $0.38 \mathrm{~m}$ \\
Width & $0.04 \mathrm{~m}$ \\
Section & $0.0002 \mathrm{~m}^{2}$ \\
Mass density & $3132 \mathrm{~kg} / \mathrm{m}^{3}$ \\
Bending stiffness & $5.3290 \mathrm{~N} \cdot \mathrm{m}^{2}$ \\
\hline \hline
\end{tabular}

for the discrete representation. In the above equations, $G(\omega)$ and $G_{d}(\omega)$ define the controller transfer functions; $\xi_{p}\left(k_{x}\right)$ and $\xi_{p}^{d}\left(k_{x}\right)$ are the $p$ th continuous and discrete modal wave-number components, respectively. In both discrete and continuous cases, they appear as the ratio of the disturbance and control path transfer functions. Substituting the above expressions for the control input in Eq. (6), the far-field pressure is then obtained using Eqs. (8) and (10).

Recalling Eq. (8), it is straightforward to show that using the far-field pressure $p(r, \theta, t)$ as error information would yield the same optimum control force as the continuous wave-number component $\widetilde{\tilde{w}}\left(k_{0} \sin \theta, t\right)$. Thus the present analysis can be seen as a comparison between the use of a discrete wave-number sensor as described in Sec. I $B$ and a microphone located in the far field.

\section{Numerical results}

Figure 2 presents the magnitude and phase of the controller transfer function versus frequency obtained for the continuous [Eq. (20)] and discrete [Eq. (21)] representations. The beam characteristics and its first six natural frequencies are given in Tables I and II. Ten modes are included in the modal series with a constant damping ratio $\left(\eta_{p}=0.01, p=1,2, \ldots, 10\right)$. The beam is surrounded by air with density $\rho=1.27 \mathrm{~kg} / \mathrm{m}^{3}$ and sound velocity $c=343$ $\mathrm{m} / \mathrm{s}$. The disturbance force is a bandlimited white noise between 0 and $650 \mathrm{~Hz}$, applied at $x_{d}=0.43 L_{x} / 2$. The piezoelectric actuator has length $2 c_{a}=0.1 L_{x} / 2$ and its center is located at $x_{c}=-0.71 L_{x} / 2$. It is assumed for brevity that the actuator characteristics yield a unity transfer function between the actuator input voltage and the resulting bending moment $M^{c}$. The same assumption applies to the other transducer models, i.e., disturbance shaker and sensor accelerometers. The wave-number component to be minimized at $k_{x}=k_{0} \sin \theta$ is associated with the direction of radiation $\theta=45^{\circ}$. The solid line corresponds to the continuous representation; the dashed, dashdot, and dotted lines correspond to the discrete representation using three-, five-, and seven-point sensors, respectively. As expected, the discrete representation becomes more accurate as the number

TABLE II. Beam analytical natural frequencies.

\begin{tabular}{cc}
\hline \hline Mode & Natural frequency (Hz) \\
\hline 1 & 31.73 \\
2 & 126.9 \\
3 & 285.6 \\
4 & 507.7 \\
5 & 793.2 \\
6 & 1142 \\
\hline \hline
\end{tabular}




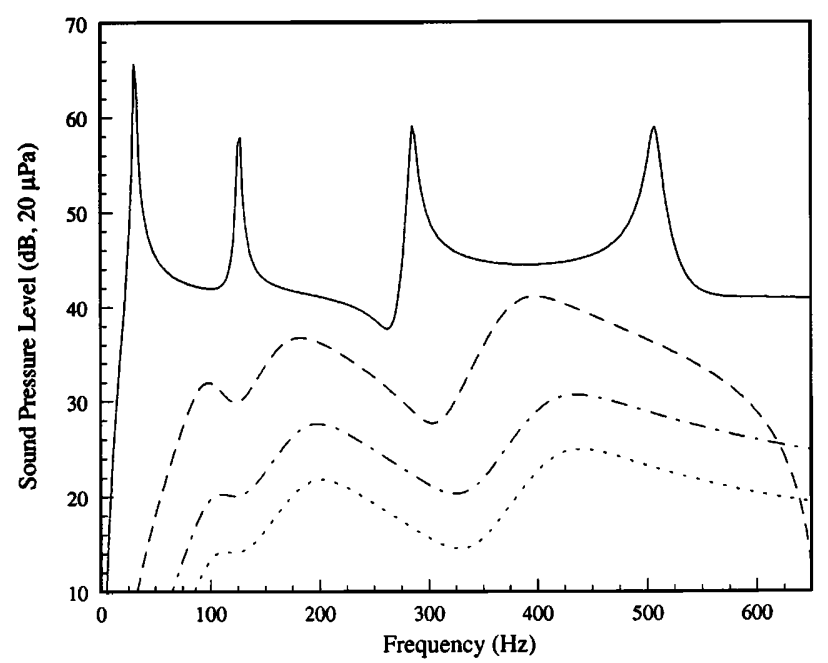

FIG. 3. Far-field pressure for in the direction of minimization: (a) $\longrightarrow$, uncontrolled system; (b),-- (c).-- , and (d)........, controlled system using three, five, and seven point sensors, respectively.

of point sensors is increased. In all the cases presented here, the error between the discrete and continuous controller transfer functions is very small at the resonant frequencies due to the fact that the wave-number component is better approximated for the simple modal shapes found on-resonance. A more detailed explanation can be found in Sec. I B 4 of the companion paper.

Figure 3 shows the influence of the errors between continuous and discrete representations on the controller performances. The modulus of the far-field pressure was computed over the frequency range $0-650 \mathrm{~Hz}$ at location $\left(r=10 L_{x}, \theta=45^{\circ}, \phi=0\right)$ for the controlled and uncontrolled system. The solid line represents the pressure for the uncontrolled system. The three dashed lines represent the pressure for the controlled system using the discrete controller with three- (dash), five- (dashdot), and seven(dot) point sensors. Note that the continuous controller gives zero pressure over the entire bandwidth at this radiation angle and thus, the corresponding curve is not shown here. The total reduction of the sound pressure level averaged over the frequency range $5-650 \mathrm{~Hz}$ is expressed in $\mathrm{dB}$ as

$$
\Delta_{\mathrm{spl}}=10 \log \left(\frac{\bar{p}(r, \theta)}{\bar{p}^{c}(r, \theta)}\right) .
$$

In the above expression, $\bar{p}(r, \theta)$ and $\bar{p}^{c}(r, \theta)$ are the meansquare pressure values of the uncontrolled and controlled system averaged over the bandwidth,

$$
\bar{p}(r, \theta)=\frac{1}{\omega_{\max }-\omega_{\min }} \int_{\omega_{\min }}^{\omega_{\max }} p(r, \theta, \omega) p^{*}(r, \theta, \omega) d \omega .
$$

In all of the three cases, the controlled system presents significant attenuation over the entire bandwidth. Accordingly to Fig. 2, the reduction is more important onresonance than off-resonance and better results are achieved with a larger number of point sensors. The total reductions averaged over $5-650 \mathrm{~Hz}$ are 13.6, 22.7, and 28.5

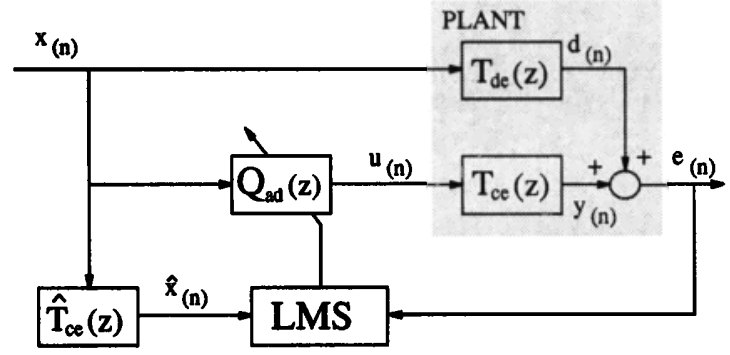

FIG. 4. Feedforward adaptive controller using the filtered-X LMS algorithm.

$\mathrm{dB}$ using three, five, and seven point sensors, respectively. Hence, good results are achieved with only a few point sensors.

Recalling Fig. 2, the phase of the controller transfer function is seen to be positive above $300 \mathrm{~Hz}$. In other words, the optimal solution in the frequency domain does not ensure causality for the controller. Therefore, the above results can not be achieved in real situations. The computer simulations described in the next sections will show more realistic results by providing a time domain optimal solution for the controller transfer function.

\section{FEEDFORWARD ADAPTIVE CONTROL IMPLEMENTATION}

The sensing technique is next implemented in a feedforward adaptive control structure using the filtered-X LMS algorithm. ${ }^{9}$ The algorithm is first presented. Then, the design of the filter array is briefly outlined. Finally, the time domain simulation procedure is described for the single channel controller applied to a simply supported beam.

\section{A. Filtered-X LMS algorithm}

A conceptual schematic of the single channel feedforward adaptive controller using the filtered- $\mathrm{X}$ algorithm is shown in Fig. 4. The plant represents the structural acoustic system, i.e., the inputs are structural excitations (actuators) and the outputs are related to acoustic radiation (sensors). For the SISO system presented here, the plant output $e(n)$ at time $t_{n}$ is the combination of the response due to the disturbance input $x(n)$ and the control input $u(n)$. This relation can be expressed in the $z$ domain as

$$
E(z)=D(z)+Y(z)=D(z)+T_{c e}(z) U(z),
$$

where the upper case letters refer to the $z$ transform of the associated sequence represented with lower case letters. Here, $T_{c e}(z)$ denotes the $z$ transform of the transfer function between the control input and the resulting sensor output. In a feedforward adaptive structure, the control sequence $u(n)$ is obtained by filtering a reference signal that is coherent to the disturbance $x(n)$ through an adaptive FIR filter $Q_{a d}(z)$, also referred to as compensator,

$$
U(z)=Q_{a d}(z) X(z) .
$$

Taking the inverse $z$ transform of Eq. (25), the control input becomes in the time domain, 


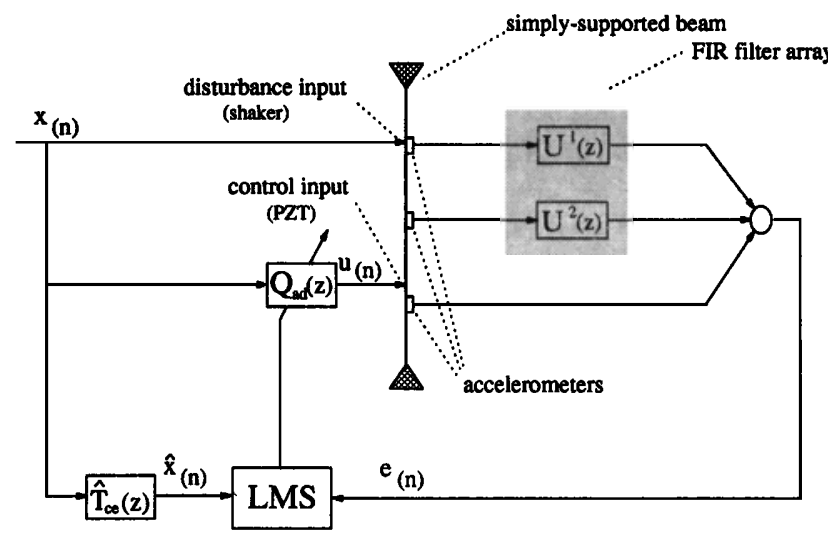

FIG. 5. Sensing configuration applied to the radiation control of a simply supported beam.

$$
u(n)=\sum_{l=0}^{N_{a d-1}} Q z^{-l} x(n),
$$

where $z^{-l}$ denotes a time delay of $l$ samples, i.e., $z^{-l} x(n)=x(n-l)$, and $Q_{l}\left(l=0, \ldots, N_{a d}-1\right)$, the compensator coefficients. Substituting Eq. (25) in (24), the error signal becomes

$$
E(z)=D(z)+T_{c e}(z) Q_{a d}(z) X(z) .
$$

The LMS algorithm adapts the coefficients $Q_{l}$ in order to minimize the cost function $J=E\left\{e^{2}(n)\right\}$, where $E$ denotes the expectation operator. Since this error function is quadratic with respect to the adaptive filter coefficients, only one minimum exists and the steepest descent method can be used to update the coefficients,

$$
Q_{l}(n+1)=Q_{l}(n)-\mu \frac{\partial J}{\partial Q_{l}}, \quad l=0,1, \ldots, N_{a d}-1,
$$

$\mu$ is the convergence parameter that controls the stability of the algorithm. The time domain LMS algorithm uses an instantaneous gradient approximation to estimate $\partial J / \partial Q_{l}$ at time $t_{n}$. Substituting $J$ in the above expression and removing the expectation operator yields

$$
\begin{gathered}
Q_{l}(n+1)=Q_{l}(n)-2 \mu e(n) \hat{x}(n-l), \\
l=0,1, \ldots, N_{a d}-1,
\end{gathered}
$$

where $\hat{x}(n)$ is the filtered-X signal. It is defined in the $z$ domain by $\hat{X}(z)=\hat{T}_{c e}(z) X(z)$. The $z$-domain transfer function $\hat{T}_{c e}(z)$ represents an estimate of the actual control path $T_{c e}(z)$ and can be modeled by an infinite impulse response (IIR) filter in case of broadband disturbance as discussed in Ref. 10.

Figure 5 presents the feedforward adaptive controller along with the structural acoustic sensor applied to a simply supported beam. The sensor is composed of three accelerometers and two FIR filters. This configuration gives a good estimate of the supersonic wave-number component associated with one direction of radiation for frequencies up to the third bending mode of the beam. A shaker modeled by a point force provides the disturbance input and a single PZT piezoelectric patch modelled by a pair of moments is used as the control input.

\section{B. Filter array design}

This section outlines the design of the FIR filters implemented in the sensor. Practically, it involves the calculation of discrete impulse response coefficients such that the modeled frequency response functions match the desired transfer functions in Eq. (17). Two design methods have been introduced in the companion paper. One uses frequency domain design algorithms and the other implements optimal filtering techniques that allow the filters to be design from experimental measurements in the discrete time domain. The first design method is discussed here. It implements the Matlab function invfreqz (software by MathWorks $\left.{ }^{11}\right)$.

From Eq. (15), the sensor output sequence is expressed in the $z$ domain as

$$
E(z)=\sum_{i=1, i \neq i_{0}}^{N_{d}} U^{i}(z) X_{i}(z)+X_{i_{0}}(z),
$$

where $X^{i}(z)$ denotes the $z$ transform of the structural acceleration measured at location $x_{i}$ and $i_{0}$ refers to the direct path (unity transfer function). Here, $U^{i}(z)$ is the $z$ transform of the finite impulse response for the $i$ th filter modeling $\bar{H}_{i}(\omega)$ in Eq. (17),

$$
U^{i}(z)=U_{0}^{i}+U_{1}^{i} z^{-1}+\cdots+U_{N}^{i} z^{-N_{i}}
$$

In the above expression, $U_{0}^{i}, U_{1}^{i}, \ldots, U_{N^{i}}^{i}$ are the $N_{i}+1$ coefficients and $N_{i}$ the order of the $i$ th filter. The modeled frequency response functions $H_{i}^{m}(\omega)$ can be obtained from $U^{i}(z)$ as

$$
H_{i}^{m}(\omega)=U^{i}\left(e^{j \omega T_{s}}\right)=\sum_{l=0}^{N_{i}} U^{i} e^{-j \omega l T_{s}}, \quad i=1,2, \ldots, N_{d},
$$

where $T_{s}$ is the sampling period.

\section{Discrete time-domain computer simulation}

Unlike simulation in the frequency domain that give the optimal control performance (see Sec. I C), a discrete time domain simulation provides more realistic results as it implements the convergence process of the LMS algorithm and ensures causality for the controller. This constraint is a result of the use of physically realizable causal digital filters which cannot model acausal transfer functions. In the frequency domain, the optimal control force is readily obtained using the analytical structural and acoustic responses of the beam as shown in the first section. On the other hand, time domain control simulations require discrete transfer functions expressed in the $z$ domain to provide the beam response in the discrete time domain; in other words, the transient as well as steady state response of the beam is modelled. In order to do this, the beam response is described over the frequency range of interest by using appropriate impulse responses in discrete form as filters.

Figure 6 shows a block diagram of the simulated system corresponding to the configuration of Fig. 5. The discrete transfer functions $T_{d}^{i}(z)$ and $T_{c}^{i}(z)\left(i=1,2, \ldots, N_{d}\right)$ 


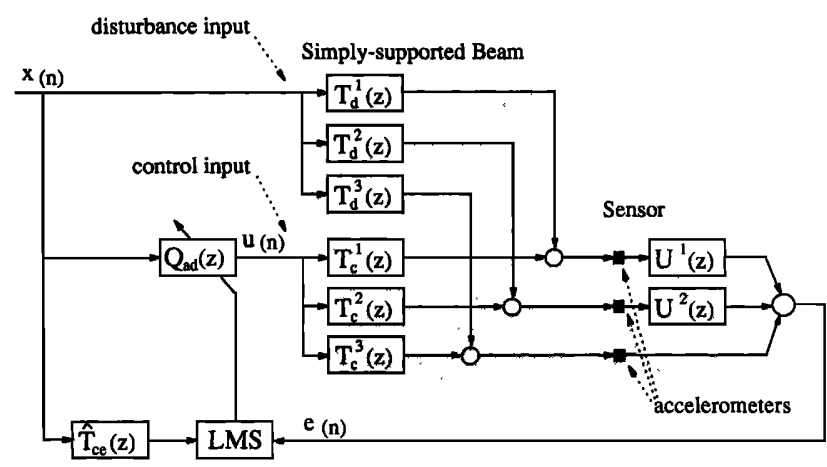

FIG. 6. Simulated system.

model the path between the disturbance input $x(n)$, at location $x_{d}$, and the beam acceleration response, at locations $x_{i}$, and the path between the control input $u(n)$, at location $x_{c}$, and the same acceleration signals. They are obtained as follows.

Recalling Eqs. (1)-(7), the analytical transfer functions between both disturbance and control inputs and the beam acceleration response at $x=x_{a}, H_{d e}\left(x_{d}, x_{a}, \omega\right)$ and $H_{c e}\left(x_{c}, x_{a}, \omega\right)$, respectively, can be approximated over the bandwidth of interest by the finite summations

$$
\begin{aligned}
H_{d e}\left(x_{d}, x_{a}, \omega\right)= & \sum_{p=1}^{p_{\max }} \psi_{p}\left(x_{d}\right) \psi_{p}\left(x_{a}\right) H_{a, p}(\omega), \\
H_{c e}\left(x_{c}, x_{a}, \omega\right)= & \sum_{p=1}^{p_{\max }} \frac{d}{d x}\left\{\psi_{p}\left(x_{c}+c_{a}\right)\right. \\
& \left.-\psi_{p}\left(x_{c}-c_{a}\right)\right\} \psi_{p}\left(x_{a}\right) H_{a, p}(\omega),
\end{aligned}
$$

where $p_{\max }$ is the number of modes included in the representation. Here, $H_{a, p}(\omega)$ is the acceleration frequency response of the $p$ th mode defined as

$$
H_{a, p}(\omega)=\frac{-\omega^{2}}{\omega_{p}^{2}-\omega^{2}+2 j \eta_{p} \omega_{p} \omega}
$$

Using a ramp invariance approximation, the above secondorder modal contribution has the following $z$-domain representation, ${ }^{12}$

$$
\begin{aligned}
& H_{a, p}^{d}(z) \\
& =\frac{\alpha z^{2}-2 \alpha z+\alpha}{z^{2}-2 z \exp \left(-\eta_{p} \omega_{p} T_{s}\right) \cos \left(\omega_{p, d} T_{s}\right)+\exp \left(-2 \eta_{p} \omega_{p} T_{s}\right)},
\end{aligned}
$$

where

$$
\alpha=\frac{\sin \left(\omega_{p, d} T_{s}\right)}{\omega_{p, d} T_{s}} \exp \left(-\eta_{p} \omega_{p} T_{s}\right)
$$

and $\omega_{p, d}=\omega_{p} \sqrt{1-\eta_{p}^{2}}$. Here, $T_{s}=1 / F_{s}$ denotes the discrete time sampling period. Note that the above discrete transfer function has poles inside the unit circle which ensures stability. Equation (36) can now be substituted in Eqs. (33) and (34) to obtain the discrete transfer functions for the beam:

$$
\begin{aligned}
H_{d e}^{d}\left(x_{d}, x_{a}, z\right)= & \sum_{p=1}^{p_{\max }} \psi_{p}\left(x_{d}\right) \psi_{p}\left(x_{a}\right) H_{a, p}^{d}(z), \\
H_{c e}^{d}\left(x_{c}, x_{a}, z\right)= & \sum_{p=1}^{p_{\max }} \frac{d}{d x}\left\{\psi_{p}\left(x_{c}+c\right)\right. \\
& \left.-\psi_{p}\left(x_{c}-c\right)\right\} \psi_{p}\left(x_{a}\right) H_{a, p}^{d}(z) .
\end{aligned}
$$

The above expressions can be simplified into a rational transfer function of the form $A(z) /(1-B(z))$, where $A(z)$ and $B(z)$ are obtained by convolving the second-order polynomials in $z$ of Eq. (36). The coefficients of $T_{d}^{i}(z)$, $T_{c}^{i}(z)\left(i=1,2, \ldots, N_{d}\right)$ are then calculated substituting the input force locations, i.e., $x_{d}$ and $x_{c}$, respectively, and the point sensor locations, i.e., $x_{i}, i=1,2, \ldots, N_{d}$, in Eqs. (38) and (39).

The estimated filtered-X control path $\hat{T}_{c e}(z)$ is constructed from the analytical transfer function between the control force and the sensor output,

$$
\hat{H}_{c e}(\omega)=\sum_{i=1, i \neq i_{0}}^{N_{d}} H_{c e}\left(x_{c}, x_{i}, \omega\right) \bar{H}_{i}(\omega)+H_{c e}\left(x_{c}, x_{i_{0}}, \omega\right) .
$$

In the above expression, $H_{c e}\left(x_{c}, x_{i}, \omega\right)$ represents the transfer function between the control input force and the beam acceleration at location $x=x_{i}$ [Eq. (34)], while $\bar{H}_{i}(\omega)$ is the frequency response of the $i$ th radiation filter [Eq. (17)]. The resulting transfer function, $\hat{H}_{c e}(\omega)$, is modeled by an IIR filter whose coefficients are computed using the Matlab function invfreqz.

Another way to design the IIR filter modeling the control path would be to use the beam and radiation filter discrete impulse responses, i.e., $T_{c}^{i}(z)$ and $U^{i}(z)$, as follows:

$$
\hat{T}_{c e}(z)=\sum_{i=1, i \neq i_{0}}^{N_{d}} T_{c}^{i}(z) U^{i}(z)+T_{c}^{i_{0}}(z) .
$$

This method gives an exact filtered-X path since it is based on the actual simulated system which has discrete sampling. On the other hand, the method chosen here, based on the analytical continuous representation [Eq. (40)], is not exact. This is due to differences between the beam continuous and discrete representations (ramp invariance approximation) as well as between the analytical radiation transfer functions $\bar{H}_{i}(\omega)$ and the actual filter responses. However, when dealing with real structures and broadband excitation, the filtered-X path modeling also results in errors due to pole instability. ${ }^{10}$ Consequently, the first representation appears to be more realistic.

At this point, an important aspect of structural acoustic sensing should be noted. As the sensor is based on wave-number information, the radiation filters do not include the acoustic path time delay $r / c$. It follows that the above filtered-X path has a much smaller group delay [Eq. (16)], i.e., smoother phase, than the transfer function obtained with a microphone in the far field. In consequence, the order of $\hat{T}_{c e}(z)$ is greatly reduced and better accuracy is achieved for the filtered-X path which is a critical factor in terms of control performances. ${ }^{10}$ 
The computer simulation is carried out by performing the following sequence of computation during each step through time: (a) compute the disturbance input $x(n)$ and the control input $u(n)$ applied to the beam; (b) compute the sensor output $e(n)$; (c) compute the filtered-X input $\hat{x}(n)$; (d) update the adaptive filter weights according to Eq. (29).

\section{SIMULATION RESULTS}

The structural acoustic sensing technique is illustrated through computer simulations on sound radiation control for the simply supported baffled beam considered previously. Results are provided for the single channel filtered-X LMS control approach using a band limited broadband disturbance.

\section{A. System characteristics}

The beam and surrounding medium have the characteristics presented in Sec. I C. The reference signal is obtained in the discrete time by convolving at each time step a Gaussian white noise with the discrete impulse response $Q_{l p}(z)$ of a low-pass FIR filter. The filter cut-off frequency is $F_{c}=600 \mathrm{~Hz}$ which allows excitation of the first four bending modes of the beam (see Table II). The resulting band-limited broadband signal is used to model the point force disturbance at location $x=x_{d}$. As presented earlier, the control input models the excitation of a piezoelectric actuator at location $x=x_{c}$. It is obtained by filtering the reference signal through an adaptive FIR compensator with a 25 coefficient impulse response $\left(N_{a d}=25\right)$. The sampling frequency is $F_{s}=2000 \mathrm{~Hz}$.

Two different sensing configurations are simulated. The first uses $N_{d}=3$ accelerometers and the second uses $N_{d}=5$ accelerometers. In both cases, the $N_{d}-1$ radiation FIR filters have three coefficients and the sensor output provides an estimate of the wavenumber component evaluated in the direction $\left(\theta=45^{\circ}, \phi=0^{\circ}\right)$. A 15-order IIR filter is used to model the filtered-X path.

\section{B. Sensor output}

Figure 7 presents the structural acoustic sensor output $e(n)$ in the discrete time domain before and after control. This case corresponds to the three point sensor. The sensor output sequence before control [curve (a)] is simply obtained by running the simulation code through a number of iterations with the weights of the compensator $Q_{a d}(z)$ being set to zero. The next step consists of updating the weights of the compensator until convergence has occurred. The sensor output sequence [curve (b)] after control is then obtained by freezing the weights of the compensator. The dominance of the first mode of the beam clearly appears on the time history before control. The wave-number component of this mode, evaluated in the direction of minimization (supersonic region) presents a large magnitude, which explains its high radiation efficiency. About 200000 iterations were required to reach quasistationarity for the mean-square value of the minimized error signal. A larger value of the convergence pa-
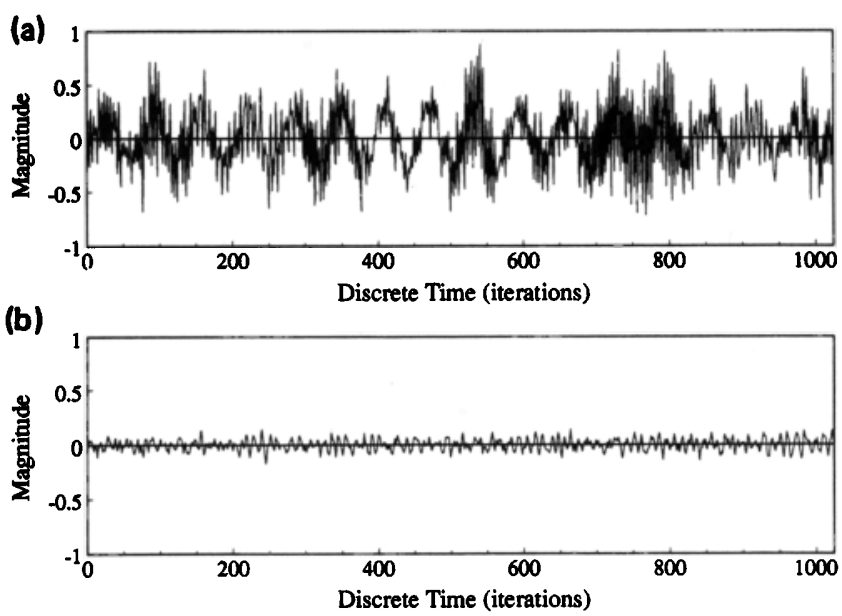

FIG. 7. Structural acoustic sensor output time history before (a) and after (b) control $\left(N_{d}=3\right)$.

rameter $\mu$ would result in faster convergence, but stability aspects must be considered. The mean-square value of the sensor output time history before and after control was calculated over 204800 points. A band averaged reduction of $13.7 \mathrm{~dB}$ is achieved. The second case, i.e., $N_{d}=5$ point sensor, gives a band averaged reduction of $11.5 \mathrm{~dB}$. These results are now further analysed in the frequency domain.

The sensor output autospectra, shown in Fig. 8 along with the autospectrum of the disturbance input $x(n)$, are computed from the DFT of the discrete time signals before and after control using $\mathbf{5 0}$ averages. As expected, the disturbance input (dashed line) is a white noise over the bandwidth 0-600 $\mathrm{Hz}$ where the filter cuts in. The sensor output autospectra before control contains the first four modes of the modeled beam. The dominance of the first mode noticed on the time history before control also appears in the frequency domain. After control, the error signal frequency content is significantly reduced over the entire frequency range. Some spill over is noticed offresonance between the second and third mode resonance

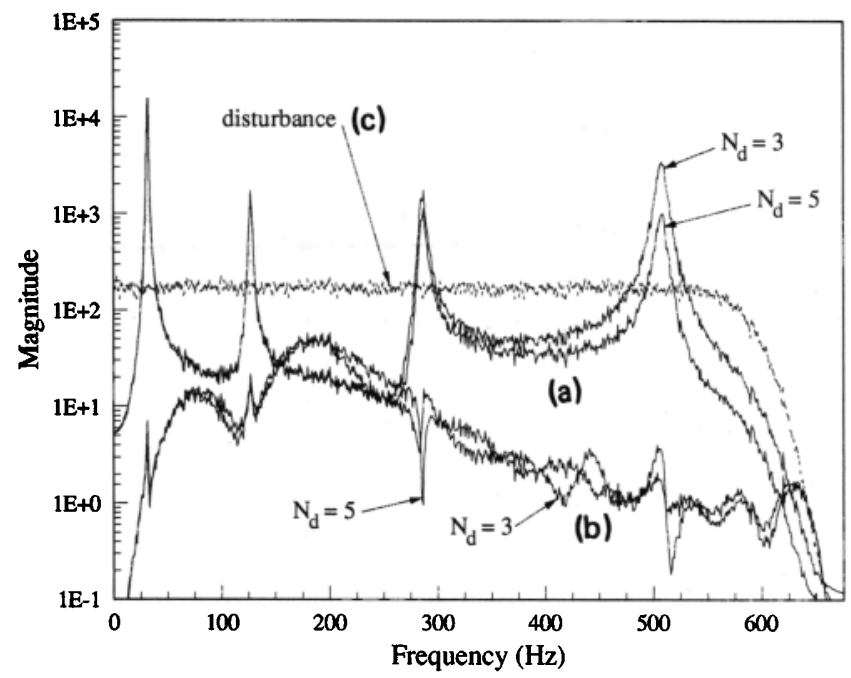

FIG. 8. Structural acoustic sensor output before (a) and after (b) control, and disturbance input (c) auto spectra. 
point. The influence of the number of structural measurements $N_{d}$ is noticeable above the third mode resonant frequency. The error between the actual sensor output (discrete wave-number component) and the desired signal (continuous wave-number component) is undoubtedly reduced using five measurement points instead of three points. As seen from the two solid lines, the three-point sensor tends to increase the estimate of the wave-number component magnitude before control. After control, the variations between the two sensor configurations also appear before the third mode resonance point. However, the tendency noticed before control, i.e., increase of the magnitude, is replaced by a more complex behavior. Globally, both cases result in the same frequency content after control. It follows that the three-point sensor gives more attenuation of the error signal than the five-point sensor above the third mode resonant frequency. Consequently, the overall reduction obtained for the sensor output is higher for the three-point sensor as found earlier from the time domain histories. However, it should be noted that this result only applies to the sensor output which is an estimate of the radiation information. In other words, it is necessary to analyze the far-field radiation before and after control in order to compare the performance of the threeand five-point sensors in terms of radiation control.

\section{Far-field radiation}

The far-field pressure information before and after control is now discussed. The calculations use the analytical expressions of Sec. I A. From the impulse response of the low-pass filter $Q_{l p}(z)$ used to generate the input band limited disturbance, the disturbance force complex amplitude $F^{d}(\omega)$ is computed over the bandwidth [Eq. (32)]. Multiplying $F^{d}(\omega)$ by the frequency response of the adaptive filter after convergence gives the control input force over the bandwidth $M^{c}(\omega)=Q_{a d}\left(e^{j \omega T_{s}}\right) F^{d}(\omega)$. Substituting the above quantities in Eqs. (6), (10), (11), and (8), the acoustic pressure in the far field is calculated for each frequency of interest along the half circle $\left(r=10 L_{x},-\pi\right)$ $2 \leqslant \theta \leqslant+\pi / 2, \phi=0$ ).

The far-field pressure spectrum in the direction of minimization at $\theta=45^{\circ}$ (Fig. 9) shows significant reduction over the entire frequency range. More than 20-dB reduction is achieved at the four resonant frequencies. The curves before (solid line) and after (dashed and dotted lines) control are relatively close from the sensor output auto-spectra shown in Fig. 8 in the lower frequency range. Again, some spill over is noticed between the second and the third mode resonance points. At higher frequencies (above the third mode frequency point), the sensor accuracy deteriorates. Consequently, the sound reduction in the far field is smaller than the reduction noticed on the sensor output. The best results are obtained with the five-point sensor due to its better accuracy. This is consistent with the results from the frequency domain analysis shown in Fig. 3.

The above results are further interpreted by analysing the compensator frequency response functions, i.e., the transfer function between the reference signal $x(n)$ and the

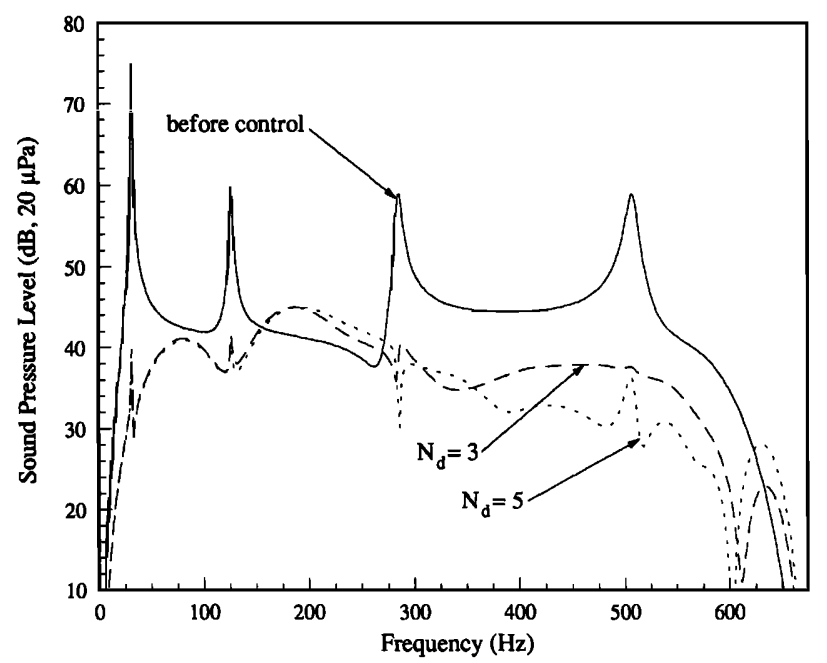

FIG. 9. Far-field pressure at $\left(\theta=45^{\circ}, \phi=0^{\circ}\right)$; (a) $\longrightarrow$, before control; (b) - - and (c) ........, after control with $N_{d}=3$ and $N_{d}=5$, respectively.

control input $u(n)$, shown in Fig. 10. The solid line represents the optimal transfer function found from Eq. (20). As mentioned in Sec. I C, this response results in zero pressure at $\theta=45^{\circ}$ over the frequency range. The dashed and dotted lines represents the actual compensator frequency response functions using the three- and five-point sensors, respectively. They are obtained from the coefficients of the adaptive filter after convergence using Eq. (32). In both cases, significant variations between the optimal [curve (a)] and actual [curves (b) and (c)] responses are noticed. As expected, the frequency response function of the compensator after convergence is directly related to the control performances. The frequencies at which some spill-over was noticed in Fig. 9, i.e., between 150 and $260 \mathrm{~Hz}$, correspond effectively to the largest variations in Fig. 10. On the other hand, the notch of the far-field pressure noticed around $600 \mathrm{~Hz}$ in Fig. 9 is explained by the coincidence at that frequency of the optimal

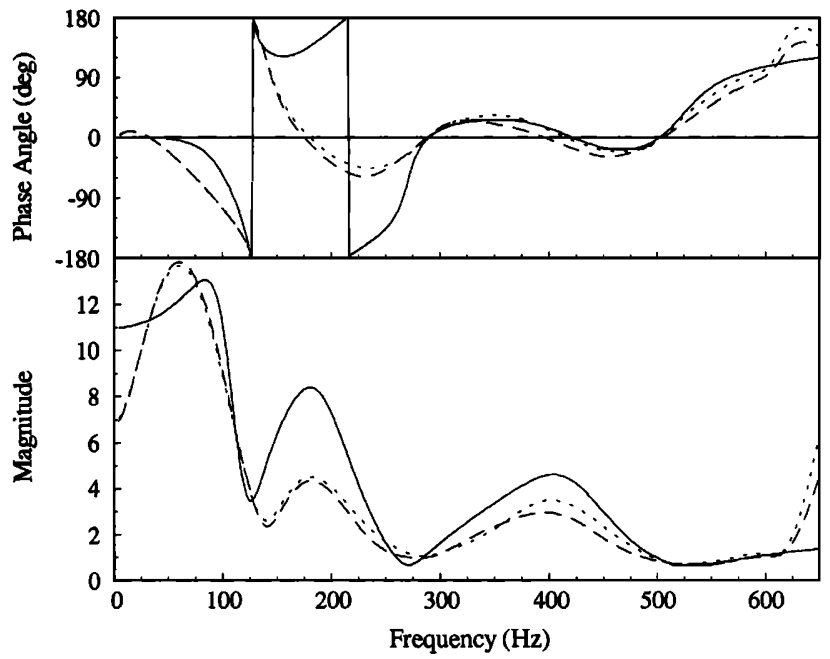

FIG. 10. Compensator frequency response function; (a) - optimal response; (b) --- and (c)......., actual response with $N_{d}=3$ and $N_{d}=5$, respectively. 


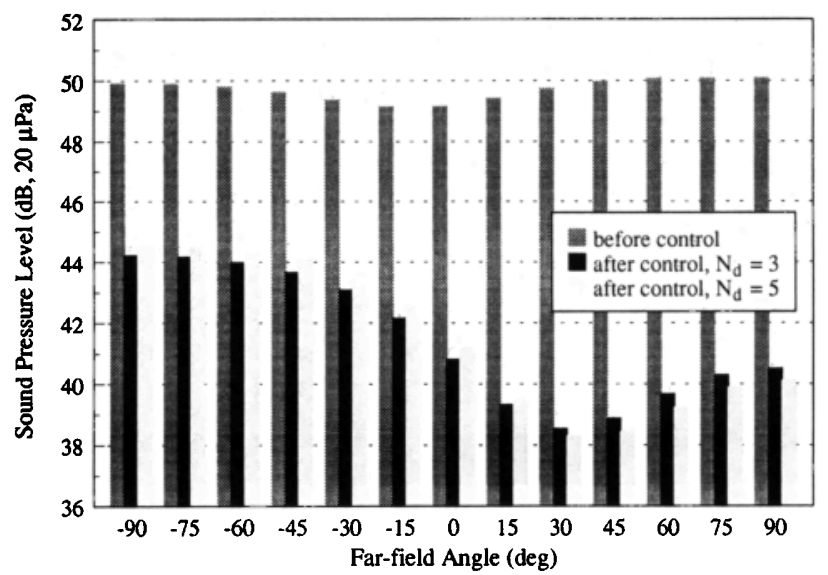

FIG. 11. Total sound pressure level averaged over $5-650 \mathrm{~Hz}$ before and after control with $N_{d}=3$ and $N_{d}=5$, respectively.

and actual frequency response functions. Moreover, the five-point sensor (dotted line) yields a control transfer function closer to the optimal solution than the three-point sensor (dashed line), especially above the third resonant frequency. This results in larger attenuations at the corresponding frequencies.

The variations between the optimal and actual responses are now further explained. Recalling the discussion of Sec. I C, the optimal solution does not ensure causality. For a system to be causal, the disturbance path must present a longer time propagation delay than the control path. If the above requirement is met over the frequency range of interest, the optimal frequency response of the compensator, defined as the ratio of the disturbance path transfer function over the control path transfer function [see Eq. (20)], has a negative phase response at all frequencies of interest and thus, can be modeled by a digital filter. In the present case, the system appears to be acausal above $300 \mathrm{~Hz}$, i.e., the optimal phase response becomes positive above this frequency [see Fig. 10, curve (a)]. Therefore, the controller compensator is unable to match the optimal response over the entire frequency range, even with an infinite number of coefficients. Consequently, the time domain simulation results in Fig. 9 present smaller attenuation than the corresponding frequency domain results shown in Fig. 3, mainly due to the causality issue discussed above. As seen from Fig. 10, the phase response of the simulated controller after convergence decreases between 120 and $240 \mathrm{~Hz}$ whereas the optimal phase response is increasing in the same bandwidth. As mentioned earlier, this phase shift results in spill-over in the frequency range 120-240 Hz. Above $300 \mathrm{~Hz}$, the controller response follows the optimal response with a $360^{\circ}$ phase shift that gives good attenuation. One way to make the system causal over the complete frequency range is to add a time delay in the disturbance path. However this solution is often impractical. Burdisso et al. have developed analytical formulations to predict reduction in attenuation as the system becomes more acausal. ${ }^{13}$

Figure 11 presents the total sound pressure level aver- aged over the frequency range $5-650 \mathrm{~Hz}$ versus the radiation angle $\theta$,

$$
I_{\text {spl }}(\theta)=10 \log \left(\frac{\bar{p}(\theta)}{2 p_{\text {ref }}^{2}}\right),
$$

where $\bar{p}(\theta)$ represents the mean-square pressure value at angle $\theta$ averaged over the bandwidth [Eq. (23)]. Global control is achieved over the entire frequency range. The plot shows a reduction of 11 and $11.5 \mathrm{~dB}$ in the direction of minimization $\left(\theta=45^{\circ}\right)$ for the three-point and five-point sensor, respectively. This result is consistent with the overall reduction obtained from the sensor output $(13.7 \mathrm{~dB}$ for $N_{d}=3$ and $11.5 \mathrm{~dB}$ for $N_{d}=5$ ). Again, note that the five point sensor output gives a more accurate far-field information than the three-point sensor, which yields greater far-field attenuation.

\section{CONCLUSIONS}

A real time structural acoustic sensor has been developed for application to active control of sound radiated by rectangular vibrating surfaces. The case of a simply supported baffled beam is considered in this paper. The time domain filtered-X LMS algorithm is implemented to achieve control over a broadband frequency range. Computer simulations show the ability of the technique to replace the use of error microphones in the far field.

For the low-order modes of the beam, only a few point sensors are needed to measure the structural information. Moreover, the FIR filters that process the discretized structural response to provide radiation information have a small number of coefficients due to the constant magnitude and linear phase of the free-space Green's function and the stationary cost function used in feedforward control. The resulting error signal is a fairly good approximation of the supersonic wave-number component associated with a prescribed direction of radiation. Moreover, the computer simulations show that the errors introduced by structural acoustic sensing in the radiation information are not critical in feedforward control compared to other issues such as causality and control authority. Therefore, the proposed structural acoustic sensor presents a important alternative to the use of distributive structural sensors (PVDF films) in ASAC approaches since it provides broadband radiation information.

\section{ACKNOWLEDGMENTS}

The authors gratefully acknowledge the support of this work by the Office of Naval Research under Grant No. ONR-N0004-92-j-1170-P00007.

${ }^{1}$ C. R. Fuller, "Active control of sound transmission/radiation from elastic plates by vibration inputs I. Analysis," J. Sound Vib. 136, 1-15 (1990).

${ }^{2}$ C. R. Fuller, R. J. Silcox, V. L. Metcalf, and D. E. Brown, "Experiments on structural control of sound transmitted through an elastic plate," in Proceedings of American Control Conference, edited by $\mathbf{H}$. Vincon Poor (Pittsburgh, PA, 1989), pp. 2079-2089.

${ }^{3}$ R. L. Clark and C. R. Fuller, "Modal sensing of efficient radiators with PVDF distributed sensors in active structural acoustic approaches," J. Acoust. Soc. Am. 91, 3321-3329 (1992). 
${ }^{4}$ C. R. Fuller and R. A. Burdisso, "A wavenumber domain approach to the active control of structure-borne sound," J. Sound Vib. 148(2), 355-360 (1991).

${ }^{5}$ J. P. Maillard and C. R. Fuller, "Advanced time domain sensing techniques for structural acoustic systems. I. Theory and design," J. Acoust. Soc. Am. 95, 3252-3261 (1994).

${ }^{6}$ S. J. Elliott, I. M. Stothers, and P. A. Nelson, "A Multiple Error LMS Algorithm and Its Application to the Active Control of Sound and Vibration," IEEE Trans. Acoust. Speech Signal Process. ASSP-35 (10), 1423-1434 (1987).

${ }^{7}$ R. L. Clark, C. R. Fuller, and A. L. Wicks, "Characterization of multiple piezoelectric actuators for structural excitation," J. Acoust. Soc. Am. 90, 346-357 (1991).
${ }^{8}$ M. C. Junger and D. Feit, Sound, Structures and Their Interaction (MIT, Boston, 1986).

${ }^{9}$ B. J. Widrow and S. D. Stearns, Adaptive Signal Processing (PrenticeHall, Englewood Cliffs, NJ, 1986).

${ }^{10} \mathrm{~J}$. S. Vipperman, R. A. Burdisso, and C. R. Fuller, "Active control of broadband structural vibrations using the LMS adaptive algorithm," J. Sound Vib. 166(2), 283-299 (1993).

${ }^{11}$ PC-Matlab for MD-DOS Computers (The MathWorks, Inc., South Natick, MA, 1989).

${ }^{12}$ K. J. Åström and B. Wittenmark, Computer-controlled Systems (Prentice-Hall, Englewood Cliffs, NJ, 1990).

${ }^{13}$ R. A. Burdisso, J. S. Vipperman, and C. R. Fuller, "Causality analysis of feedforward-controlled systems with broadband inputs," J. Acoust. Soc. Am. 94, 234-242 (1993). 